\title{
Numerical Analysis and Experimental Verification Based on Design Parameter Variation of Vacuum-Insulated Pipe for Cryogenics
}

\author{
Jae-Hoon Lee ${ }^{1, a}$, Si-Pom Kim, b* , Geun-Ho Lee Le $^{1, c}$ \\ ${ }^{1}$ Department of Mechanical Engineering, Dong-a University, Busan City, Republic Korea \\ adhxowkd31019@naver.com, bspkim@dau.ac.kr, Igh6072@gmail.com
}

Keywords: Vacuum-insulated pipe, Below type, Joint type, Boil off gas, Transient thermal an alysis, Thermal-structural coupled field analysis.

\begin{abstract}
Since natural gas is mainly transported or supplied in a liquefied state under high pressure, BOG (boil off gas) is frequently generated due to the adiabatic relation outside the pipe. To reduce BOG, various studies are being conducted. Recently, vacuum-insulated pipes are mainly used. Thus, in this study, temperature distribution in a vacuum-insulated pipe was observed through thermal analysis with the pipe type and diameter as design parameters. Additionally, thermal analysis was verified through an experiment under the same condition. BOG was calculated based on numerical analysis verification results and the heat transfer performance was confirmed. In addition, the generated thermal stress and thermal deformation changes were observed by implementing the temperature distribution obtained through the thermal analysis in thermalstructure coupling analysis. Accordingly, the structural safety of a vacuum-insulated pipe was evaluated.
\end{abstract}

\section{Introduction}

Nowadays, the need of natural gas for energy production and its worldwide demand is gradually increasing compared to oil. Accordingly, many studies have been conducted for liquefied natural gas (LNG) transport and supply [1,2]. As LNG is liquefied at a temperature of -179 , it is transported through pipes. Consequently, BOG (boil-off gas) is frequently generated due to the adiabatic relation outside the pipe $[3,4]$. To minimize BOG, existing methods such as vacuum insulation, multi-layer insulation, and vacuum powder insulation have been used [5]. Among the existing methods, the vacuum insulation method includes the technology to prevent conduction and convection through rarefied gas. Therefore, research related to this method has been actively conducted. In particular, the vacuum insulation method has been applied to the pipes and the method using double pipes has been mainly used. This method is applied to reduce BOG by minimizing heat loss to the outside environment by vacuum through the insertion of an insulator with excellent insulation performance inside the double pipes. However, in spite of the high insulation performance, much work has not been conducted on vacuum-insulated pipes through insulation using double pipes and vacuum operation $[6,7]$.

As the main design parameters, various design factors such as various types of vacuum-insulated pipes, vacuum layer thickness, vacuum strength, insulation thickness, insulator material, and insulator density (number of layers per length) affect the heat transfer of a vacuum-insulated pipe [8]. However, basic data and research related to these parameters has not been clearly presented until now.

In this study, temperature distribution was observed through thermal analysis based on the variation in the type and diameter of a vacuum-insulated pipe for a cryogenic environment and thermal analysis was verified through experiments under the same condition. BOG was calculated based on numerical analysis verification results and the heat transfer performance was confirmed. In addition, the generated thermal stress and thermal deformation changes were observed by implementing the temperature distribution obtained via the thermal analysis in thermal-structure coupling analysis to evaluate the structural safety of the vacuum insulated pipe. 


\section{Numerical Analysis}

Finite Element Model. A finite element model was constructed using the pipe shape, which was shrunk to $1 / 16$ to reduce the time required for numerical analysis. The types applied in numerical analysis are shown in Fig. 1 and Table 1, respectively.

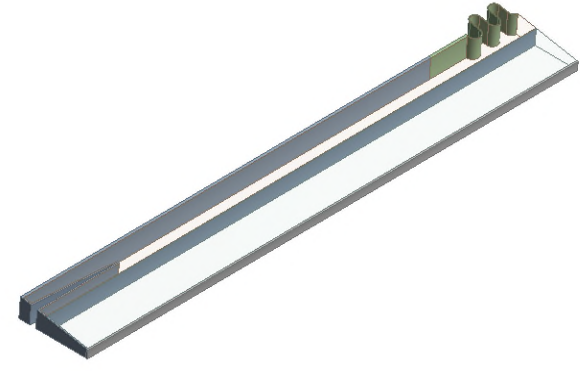

(a) below type

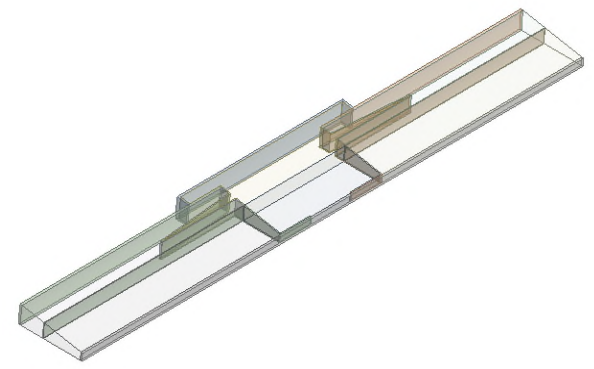

(b) joint type

Fig. 1 Type of vacuum-insulated pipe.

Table 1. Main design parameters of vacuum-insulated pipe.

\begin{tabular}{|l|l|}
\hline Vacuum-insulated pipe type & bellows, joint \\
\hline pipe length $(\mathrm{mm})$ & 2000 \\
\hline pipe radiation $(\mathrm{mm})$ & $15,50,100,150$ \\
\hline Insulation radiation $(\mathrm{mm})$ & 15 \\
\hline Insulator material & Polyurethane \\
\hline
\end{tabular}

Fig. 1 (a) shows the below type of the vacuum-insulated pipe, while Fig. 1 (b) shows the joint type of the vacuum-insulated pipe. 3D modeling was performed by classifying the vacuuminsulated pipe into 3 layers, i.e., vacuum layer, insulation layer, and atmosphere layer.

Thermal Analysis. In this study, the heat transfer performance of the vacuum-insulated pipe for cryogenic environment was evaluated at low temperature. Meanwhile, thermal analysis was conducted to obtain the temperature distribution for thermal-structure coupling analysis. The boundary conditions for the analysis are shown in Table 2 .

Table 2. Boundary conditions for thermal analysis.

\begin{tabular}{|l|l|}
\hline Temperature inside the inner pipe & $-179^{\circ} \mathrm{C}$ \\
\hline Temperature outside the outer pipe & $20^{\circ} \mathrm{C}$ \\
\hline Convective heat transfer coefficient & $5 \mathrm{~W} / \mathrm{m}^{2} \mathrm{~K}$ \\
\hline Steel pipe emissivity & 0.1 \\
\hline External insulation emissivity & 0.2 \\
\hline
\end{tabular}

Since the time was varied during the actual vacuum-insulated pipe operation, transient thermal analysis was more suitable for the thermal analysis than steady-state thermal analysis. Hence, thermal analysis was conducted using transient thermal analysis. To improve the quality of the grid and shorten the time for the numerical analysis, sweep mash was employed and hexahedral grid was constructed. Sweep mash has an advantage in that a uniform grid in all areas through a specific path or rotation axis is produced. Thus, the reliability of the numerical analysis was improved. The numerical analysis was performed in a total time of $30 \mathrm{~min}$, while the transient thermal analysis was conducted at a 10 -s interval. 
Thermal-structure Coupling Analysis. The temperature distribution obtained via the transient thermal analysis was implemented in the thermal-structure coupling analysis, while the numerical analysis was conducted after applying and fixing the forced displacement constrains at both ends of the vacuum-insulated pipe. The boundary conditions for the thermal-structure coupling analysis of the vacuum-insulated pipe for analyzing the thermal stress and thermal deformation are shown in Table 3.

Table 3. Boundary conditions of thermal-structure coupling analysis.

\begin{tabular}{|l|l|}
\hline Internal pressure & 320 bar \\
\hline Vacuum pressure & $10^{-3}$ Torr $(-6.6661 \mathrm{e}-7 \mathrm{MPa})$ \\
\hline
\end{tabular}

\section{Experiment}

Temperature Measurement in Vacuum-insulated Pipe. Since it was difficult to rely on the result obtained through a simple numerical analysis process, the numerical analysis result had to be verified through an experiment. Hence, temperature data was obtained by measuring the temperature distribution of the vacuum-insulated pipe. The experimental details are shown in Fig. 2.

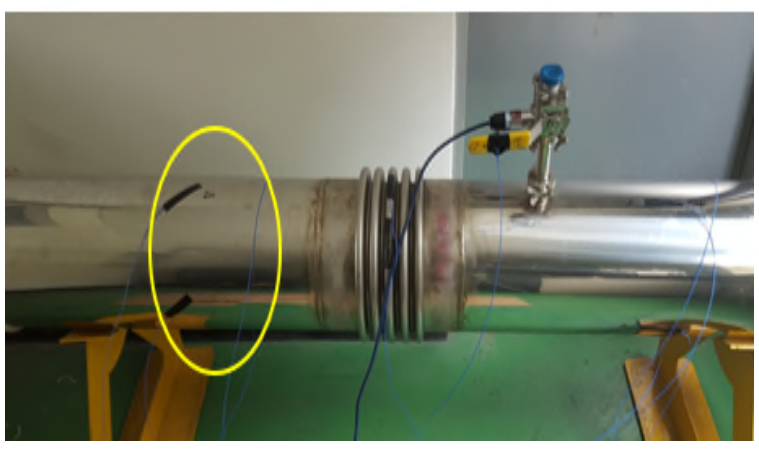

(a) Temperature measurement

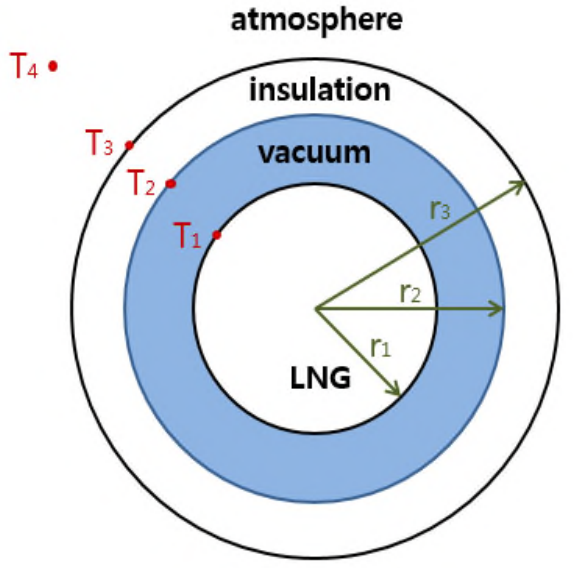

(b) Cross-section

Fig. 2 Temperature measurement of vacuum-insulated pipe for numerical analysis verification.

The actual experimental condition of the vacuum-insulated pipe is shown in Fig. 2 (a), while the cross-section of the temperature measurement is shown in Fig. 2 (b). In addition, the boundary conditions used in the experiment are presented in Table 4. To reduce the error during measurement, an adhesive thermocouple was used (9).

Table 4. Experimental boundary conditions.

\begin{tabular}{|l|l|}
\hline Measurement location & 8 points \\
\hline Thermocouple type & T-type \\
\hline Fluid & LNG \\
\hline temperature & $-179^{\circ} \mathrm{C}$ \\
\hline Convective heat transfer coefficient & $6.5( \pm 1) \mathrm{W} / \mathrm{m}^{2}{ }^{\circ} \mathrm{C}$ \\
\hline Air temperature & $22^{\circ} \mathrm{C}$ \\
\hline Operating pressure & $320 \mathrm{bar}$ \\
\hline Temperature measurement time & $30[\mathrm{~min}]$ \\
\hline
\end{tabular}


Boil-off Gas Theory. For BOG calculation, the temperature was measured at 4 locations, as shown in Fig. 2. Accordingly, BOG was calculated using equation 1 based on $T_{1}, T_{2}, T_{3}, T_{4}$ and $r_{1}, r_{2}, r_{3}$.

$$
\mathrm{q}=\frac{\mathrm{T}_{4}-\mathrm{T}_{3}}{\frac{1}{\mathrm{hA}}}=\frac{\mathrm{T}_{3}-\mathrm{T}_{2}}{\frac{\ln \mathrm{r}_{3} / \mathrm{r}_{2}}{2 \pi \mathrm{kL}}}=\frac{\mathrm{T}_{2}-\mathrm{T}_{1}}{\frac{\ln \mathrm{r}_{2} / \mathrm{r}_{1}}{2 \pi \mathrm{kL}}}=\frac{\mathrm{T}_{4}-\mathrm{T}_{1}}{\frac{1}{\mathrm{hA}}+\frac{\operatorname{lnr}_{3} / \mathrm{r}_{2}}{2 \pi \mathrm{kL}}+\frac{\operatorname{lnr}_{2} / \mathrm{r}_{1}}{2 \pi \mathrm{kL}}}
$$

T1, T2, T3, T4 : temperature

$\mathrm{r} 1, \mathrm{r} 2, \mathrm{r} 3$ : radiation

q: heat flux

h: convection

$\mathrm{k}$ : conduction

L: pipe length

\section{Numerical Analysis and Experimental Result}

Numerical Analysis Result and Experimental Verification of Temperature Distribution. The temperature values obtained through the numerical analysis were verified by comparing them with the temperature values at 8 locations, obtained via the experiment. The temperature distribution as a function of time is shown in Fig. 3.

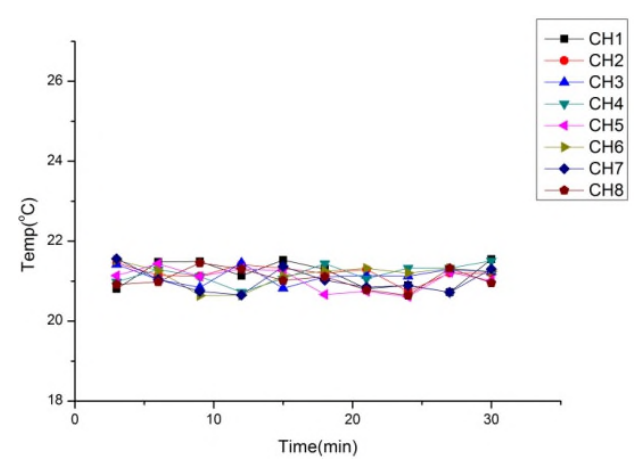

(a) result of experiment

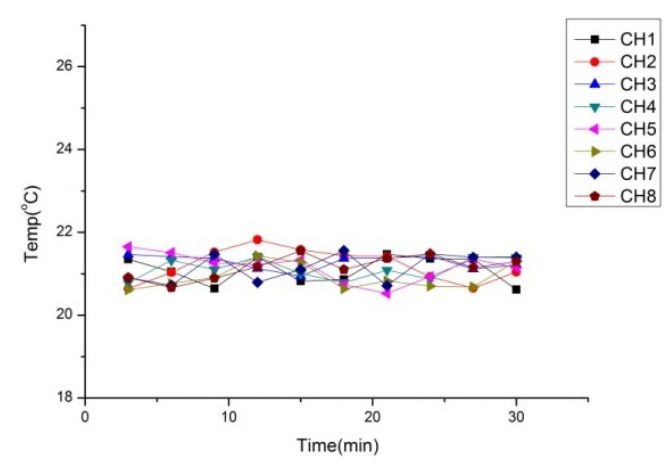

(b) result of numerical analysis

Fig. 3 Comparison of temperature distribution obtained via experiment and numerical analysis.

A slight difference of approximately $1{ }^{\circ} \mathrm{C}$ was observed between the temperature distribution results obtained via the numerical analysis and those obtained via the experiment. However, since the error value also appeared to be less than $7 \%$, it can be concluded that the numerical analysis result is reliable. Since this experiment and numerical analysis only considered the variation in diameter and type of vacuum-insulated pipe, further research on the experimental and numerical analysis approach methods using various variables is required.

\section{Structural Safety Evaluation}

BOG Result Obtained Via Thermal Analysis. Through implementation of the previous numerical analysis verification, BOG was calculated using Eq. 1; the results are shown in Fig. 4. Fig. 4 (a) shows the calculated BOG obtained using temperature distribution obtained via the numerical analysis, while Fig. 4 (b) shows the temperature distribution obtained via the experiment 


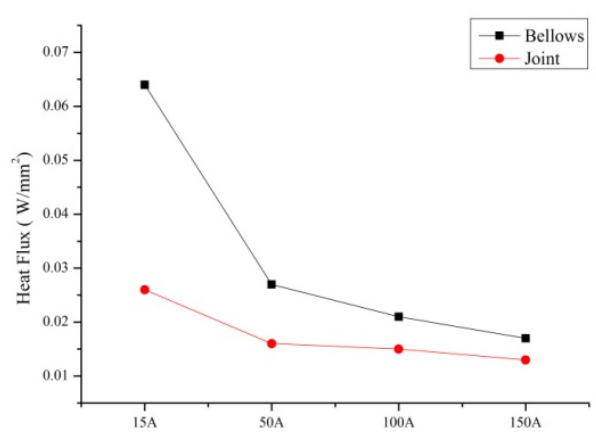

(a) Numerical analysis BOG

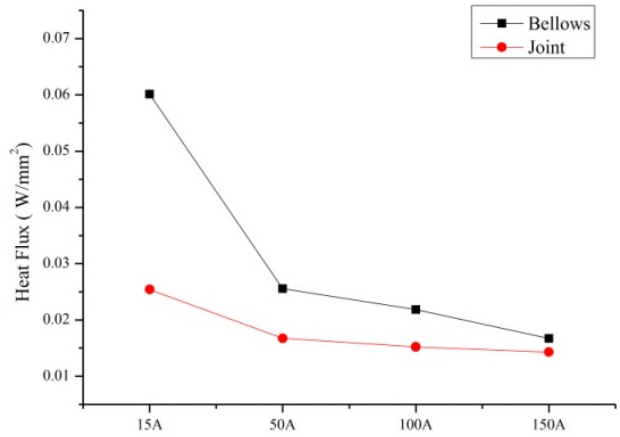

(b) Experimental BOG

Fig. 4 BOG of vacuum-insulated pipe.

According to the BOG calculation result of the vacuum-insulated pipe, it was found that the value slightly changed at different positions. However, it was confirmed that the value was less than $0.064 \mathrm{~W} / \mathrm{mm}^{2}$ for the bellows type. However, it was a very small value, confirming that external heat penetration or internal heat emission are negligible. Thus, the joint type is preferred over the bellows type when high heat insulating performance is required.

Thermal-structure Coupling Analysis Result. The temperature distribution obtained via the thermal analysis was employed to perform a numerical analysis of thermal stress and thermal deformation. Fig. 5 shows the changes in the stress and deformation as a function of diameter size. Fig. 5 (a) and (b) represent the changes in the thermal stress and thermal deformation, respectively.

The thermal-structure coupling analysis results showed that the maximum thermal stress of 228.34MPa was observed at both ends of the joint type, while thermal deformation of $0.1 \mathrm{~mm}$ occurred. For the bellows type, the maximum thermal stress of $215.52 \mathrm{MPa}$ was concentrated at the central part and the thermal deformation of $0.09 \mathrm{~mm}$ also occurred in the central pipe. This showed that the temperature of the internal fluid was cryogenic, which affected the thermal stress and thermal deformation under the actual operational condition. However, the maximum stress of 228.34 MPa in each type was much lower than the allowable stress of each material, and the deformation rate was less than $0.1 \mathrm{~mm}$, confirming the structural safety. In addition, the joint type exhibited greater stress and deformation changes than the bellows type. Excluding the heat transfer performance, it can be concluded that the bellows type is effective in structural safety-focused design. Since this is the case of a single model, additional studies are required.

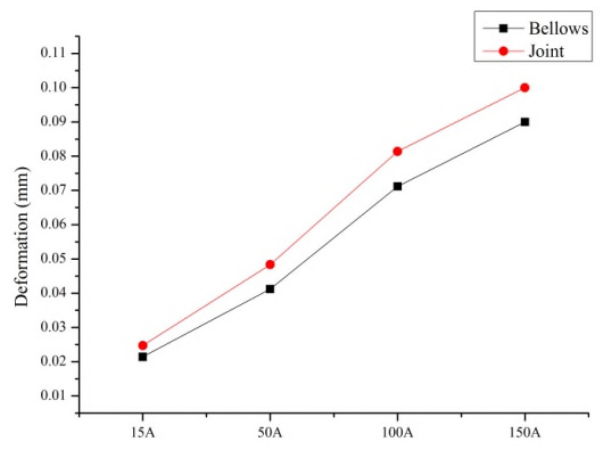

(a) stress as a function of pipe diameter

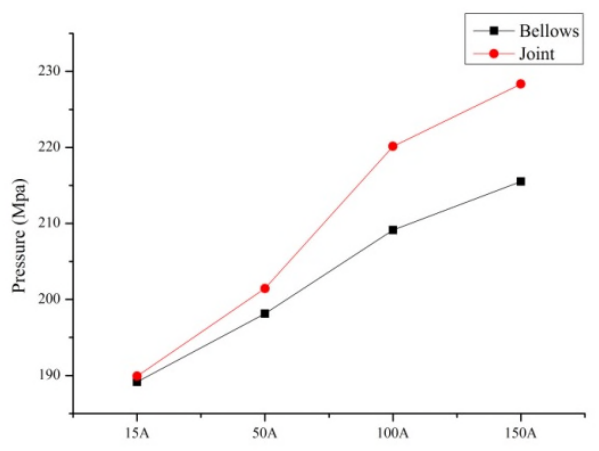

(b) deformation as a function of pipe diameter

Fig. 5 Thermal-structure coupling analysis result of vacuum-insulated pipe.

\section{Conclusions}

In this study, the verification of a numerical analysis was performed based on the temperature measurement and numerical analysis of the vacuum-insulated pipe. Based on this verification, BOG 
was investigated. The conclusions obtained from the evaluation of structural safety through the thermal-structure coupling analysis are as follows.

(1) The difference between the temperature difference results obtained via the experiment and thermal analysis for the vacuum-insulated pipe was within 5\% error. This result can be utilized as a basis for the design of a vacuum-insulated pipe through the verification of the numerical analysis.

(2) The BOG calculation results of the vacuum-insulated pipe through the thermal analysis showed that the bellows type has higher BOG that the joint type. Hence, the joint type significantly improved the thermal insulation in the design of a vacuum thermal-insulated pipe.

(3) The thermal-structure coupling analysis results showed that the joint type exhibited a stress of $228.34 \mathrm{MPa}$ and had a deformation rate $0.1 \mathrm{~mm}$ higher than that of the bellows type. However, since these values were below the yield strength of the materials, it is deemed structurally safe.

(4) In this study, only the type and diameter of the pipe are varied; however further studies are required in accordance with various design parameters, such as the variation in the pipe connection, curved part of the pipe, insulation material performance, and degree of vacuum strength.

\section{Acknowledgements}

This work was supported by the Materials and Components Technology Development Program of MOTIE/KEIT. [10046500/Development of Cryogenic $\left(-196^{\circ} \mathrm{C}\right)$, high pressure $(320 \mathrm{bar})$ welded insulation piping and valve jacket]

\section{References}

[1] D. S. Lee, A Developing Tendency of Liquefied Natural Gas Carriers, J. Korean Soc. Mar. Environ. Saf. 15(3) (2009) 269-274.

[2] C. Z. Jin, K. K. Jin, S. K. Ha, H. S. Seo, I. S. Yoon, Structure Analysis and Design optimization of Stiffeners LNG Tank, T. Korean Soc. Mech. Eng. A 36(3) (2012) 325-330.

[3] M. E. Kim, Y. T. Kim, The BOG Handling System for LNG Carriers, Korean Soc. Fluid Mach. (2005) 557-561

[4] Y. P. Lee, Y. H. Shin, S. H. Lee, K. H. Kim, Boil-Off Gas Reliquefaction System for LNG Carriers with BOG-BOG Heat Exchange, J. Soc. Naval Arch. Korea 46(4) (2009) 444-451.

[5] D. H. Lee, C. B. Jang, S. Y. Jung, J. H. Kim, H. S. Lee, B. S. Kim, J. W. Ko, Study on the Comparison of New and Used Reliquefaction System of Boil-Off-Gas by LNG Cold Energy, J. Korean Inst. Gas 14(1) (2010) 42-46.

[6] J. S. Ha, A Study on the Heat Flux Meter Location for the Performance Test of a Refrigerator Vacuum Insulation Panel, Korean J. Air-Cond. Refrig. Eng. 25(8) (2013) 471-476.

[7] H. Y. Lee, Y. M. Kim, A Study on Thermal Performance for Damaged vacuum Insulation, Architect. Inst. Korea 34(2) (2014) 319-320.

[8] H. Y. Kim, B. H. Kang, Thermal Insulation Technology for Cryogenic Cooling Devices, J. Korean Soc. Precis. Eng. 19(11) (2002) 24-30.

[9] J. W. Park, D. S. Kim, M. S. Ko, J. H. Cho, A Study on the Experimental Measurements and Its Recovery for the Rate of Boil-Off Gas from the Storage Tank of the $\mathrm{CO}_{2}$ transport Ship, Clean Technol. 20(1) (2014) 1-6. 